\title{
Natural History and Outcome of Hepatic Vascular Malformations in a Large Cohort of Patients with Hereditary Hemorrhagic Teleangiectasia
}

\author{
Elisabetta Buscarini - Gioacchino Leandro • Dario Conte • Cesare Danesino • \\ Erica Daina - Guido Manfredi - Guido Lupinacci - Gianfranco Brambilla • \\ Fernanda Menozzi · Federico De Grazia • Pietro Gazzaniga • Giuseppe Inama • \\ Roberto Bonardi · Pasquale Blotta • PierAngelo Forner • Carla Olivieri • \\ Annalisa Perna - Maurizio Grosso - Giacomo Pongiglione $\cdot$ Edoardo Boccardi • \\ Fabio Pagella $\cdot$ Giorgio Rossi $\cdot$ Alessandro Zambelli
}

Received: 7 July 2010/Accepted: 14 January 2011/Published online: 3 February 2011

(C) The Author(s) 2011. This article is published with open access at Springerlink.com

\begin{abstract}
Background Hereditary hemorrhagic telangiectasia is a genetic disease characterized by teleangiectasias involving virtually every organ. There are limited data in the literature regarding the natural history of liver vascular malformations in hemorrhagic telangiectasia and their associated morbidity and mortality.

Aim This prospective cohort study sought to assess the outcome of liver involvement in hereditary hemorrhagic telangiectasia patients.
\end{abstract}

E. Buscarini $(\varangle) \cdot$ G. Manfredi · G. Lupinacci - G. Brambilla · F. Menozzi · F. De Grazia · A. Zambelli

Gastroenterology Department, HHT Center, Maggiore Hospital,

Largo Dossena 2, 26013 Crema, Italy

e-mail: ebuscarini@rim.it

G. Leandro

Gastroenterology Unit 1, Gastroenterological Hospital

'S. De Bellis' IRCCS, Castellana Grotte, Italy

D. Conte

Gastroenterology Unit II, Fondazione IRCCS Ospedale Maggiore Policlinico, Mangiagalli e Regina Elena,

University of Milan, Milan, Italy

C. Danesino - C. Olivieri

Genetic Institute, University of Pavia, Pavia, Italy

E. Daina $\cdot$ A. Perna

Mario Negri Institute for Pharmacological Research, Clinical

Research Center for Rare Diseases "Aldo e Cele Daccò",

Bergamo, Italy

P. Gazzaniga · G. Inama

Cardiology Department, Maggiore Hospital, Crema, Italy
Methods We analyzed 16 years of surveillance data from a tertiary hereditary hemorrhagic telangiectasia referral center in Italy. We considered for inclusion in this study 502 consecutive Italian patients at risk of hereditary hemorrhagic telangiectasia who presented at the hereditary hemorrhagic telangiectasia referral center and underwent a multidisciplinary screening protocol for the diagnosis of hereditary hemorrhagic telangiectasia. Of the 502 individuals assessed in the center, 154 had hepatic vascular malformations and were the subject of the study; 198 patients

\author{
R. Bonardi \\ Radiology Department, Maggiore Hospital, Crema, Italy \\ P. Blotta $\cdot$ P. Forner \\ ENT Department, Maggiore Hospital, Crema, Italy \\ M. Grosso \\ Radiology Department, Santa Croce Hospital, \\ Cuneo, Italy \\ G. Pongiglione \\ Pediatric Cardiology Department, \\ Ospedale Pediatrico Bambin Gesù, Rome, Italy \\ E. Boccardi \\ Interventional Neuroradiology Unit, Niguarda Hospital, \\ Milan, Italy \\ F. Pagella \\ ENT Department, Policlinico S Matteo, Pavia, Italy \\ G. Rossi \\ General Surgery and Liver Transplant Unit, Fondazione IRCCS \\ Ospedale Maggiore Policlinico, Mangiagalli e Regina Elena, \\ University of Milan, Milan, Italy
}


with hereditary hemorrhagic telangiectasia and without hepatic vascular malformations were the controls. Additionally, we report the response to treatment of patients with complicated hepatic vascular malformations.

Results The 154 patients were included and followed for a median period of 44 months (range 12-181); of these, eight (5.2\%) died from VM-related complications and 39 (25.3\%) experienced complications. The average incidence rates of death and complications were 1.1 and 3.6 per 100 personyears, respectively. The median overall survival and eventfree survival after diagnosis were 175 and 90 months, respectively. The rate of complete response to therapy was $63 \%$.

Conclusions This study shows that substantial morbidity and mortality are associated with liver vascular malformations in hereditary hemorrhagic telangiectasia patients.

Keywords Hereditary hemorrhagic telangiectasia . Liver vascular malformations · Heart failure .

Portal hypertension - Gastrointestinal bleeding . Liver transplantation

\section{Introduction}

Hereditary hemorrhagic telangiectasia (HHT), or RenduOsler-Weber disease, is a genetic disorder that is characterized by widespread cutaneous, mucosal and visceral telangiectasias and is reported to affect between one and two of every 10,000 people in the general population [1-6]. The clinical presentation of HHT varies based on the number, type and location of either the telangiectasias or the larger vascular malformations (VMs). The clinical criteria for diagnosing HHT, known as the Curaçao criteria, have been established by a panel of experts [7]. Most patients with HHT have mutations in one of the two known disease-related genes: either endoglin (ENG, HHT1) [8, 9] or activin A receptor type II-like 1 (ACVRL1, HHT2) [10, 11], both of which are involved in the TGFß pathway. Mutations in the SMAD4 gene can cause the development of a rare syndrome that combines the symptoms of juvenile polyposis and HHT [12]. Recently, using linkage analysis, additional genes have been found at the predicted loci on chromosome 5 (HHT3) and on chromosome 7 (HHT4) [13, 14]. Genetic testing is available on a clinical basis.

Hepatic VMs are found in 41-74\% of HHT-affected subjects $[15,16]$. The prevalence of the hepatic VMs that are unique to HHT varies between $1 / 7,000$ and 1/12,500 and they are thus more frequent than liver involvement in either Wilson disease or other liver vascular disorders, such as Budd-Chiari. The prevalence of hepatic VMs prevalence depends substantially on which genotype of HHT is present; genotype-phenotype correlation studies have demonstrated that hepatic VMs are significantly more frequent in patients who had the HHT2 genotype than in patients who had the HHT1 genotype [17-21]. Some studies have suggested that truncating mutations have a more dramatic effect on HHT phenotype than missense mutations have on HHT phenotype [22], with a greater frequency of nosebleeds, telangiectases and pulmonary VMs found in patients who have HHT with a truncating mutation [18].

The penetrance of most of the clinical features of HHT depends on a patient's age, but the effect of age is more pronounced for hepatic VMs; according to a study of a large screening series, the mean age of patients who had hepatic VMs was 52 years old [15]; and symptoms of hepatic VMs generally do not occur before a patient reaches age 50 [18]. Previous data show a strong and significant predominance of hepatic VMs in females who have HHT, both for asymptomatic and symptomatic lesions with a male/female ratio varying from $1 / 2$ to $1 / 4.5$; therefore, the expressivity of HHT in the liver is likely dependent on a patient's sex $[18,23]$. Studies have also hypothesized that hormones modify the HHT phenotype [24].

The form of hepatic VMs ranges from small telangiectasias to large arteriovenous malformations and can diffusely involve the liver [25]. There are three types of intrahepatic shunting: hepatic artery to portal vein, hepatic artery to hepatic vein, and portal vein to hepatic vein. These three shunt types are often concomitant and can lead to the following, possibly coexistent, complications: high-output cardiac failure (HOCF), portal hypertension, biliary ischemia, encephalopathy and mesenteric ischemia [23, 25-28]. HOCF caused by hepatic AVMs may be further complicated by secondary pulmonary hypertension; when this happens, an appropriate assessment must be taken because pulmonary hypertension represents a relative contraindication to the embolization of pulmonary VMs [29].

Based on available data, pregnancy is suspected as a potential precipitating factor that leads to complications caused by hepatic VMs in HHT patients. Nonetheless, whereas a study of 262 pregnancies by 111 HHT-affected women found that $1 \%$ of the pregnancies resulted in either severe complications or death that could be attributed to pulmonary VMs [30], only nine anecdotal reports exist of severe complications occurring during pregnancy and related to hepatic VMs in HHT [31].

Thus far, data concerning the natural history of liver VMs in HHT are lacking [25, 32]. These data should ideally be derived from patients undergoing a systematic screening protocol of subjects at risk of HHT to capture the complete spectrum of hepatic VMs [3, 25, 32].

These criteria are fulfilled by the present prospective study which aimed to evaluate the outcome of liver VMs in HHT patients in terms of overall survival and event-free survival. We also evaluated responses to treatment of HHT patients who exhibited symptoms of liver VMs. 


\section{Patients and Methods}

We conducted a longitudinal, prospective cohort study at a tertiary HHT referral center. Our study considered 502 consecutive Italian patients ( 231 males, 272 females, mean age 38.7, range $3-88$, median $41 \pm 19$ ) at risk of HHT (i.e., with either a personal/familial history of nosebleeds or mucocutaneous telangiectases or visceral vascular malformations or a first degree relative affected according to the Curaçao criteria [7]). All of these patients underwent a multidisciplinary screening protocol to diagnose HHT between April 1992 and April 2007. Pertinent follow-up records until October 2008 were evaluated. The Curaçao criteria were applied to define the HHT affection status [7]. Abdominal Doppler ultrasound (US) was used to diagnose liver VMs.

\section{Inclusion Criteria}

We included patients with either clinical diagnosis of HHT (two-possible- or three-definite-Curacao criteria) or genetic diagnosis of HHT and the presence of liver VMs on Doppler US, as diffuse hepatic VMs which are unique to HHT (and with their inherent Doppler US presentation as previously described $[15,33])$.

\section{Exclusion Criteria}

We excluded subjects without HHT and patients with HHT but without liver VMs at initial screening.

\section{Control Group}

Patients with HHT without liver VMs at initial screening were used as the control group to maximize similarity between study population and controls.

\section{Screening Protocol}

Our screening protocol was approved by the Institutional Review Board. All patients (or the parents of patients $<18$ year old) who took part in the study provided written informed consent.

\section{Clinical Assessment}

Patients underwent an initial clinical assessment and periodic checkups at the referral center, according to the time schedule specified in the section Follow-up. Data collection focused on the symptoms and the clinical signs thought to be related to liver VMs and their complications. If a patient had cardiac failure we determined whether other causes of HOCF were present (e.g., thyroid disease). Each checkup included a complete physical examination and an electrocardiogram.

Complications of liver involvement were defined according to international consensus [25] and were assessed on the basis of a multidisciplinary approach including clinical, biochemical and instrumental evaluations when appropriate. Complications included the following: HOCF (with fatigue, dyspnea associated with ascites and/or limb edema; all patients were classified according to the New York Heart Association [NYHA] functional classification), complicated portal hypertension (with ascites and/or gastrointestinal bleeding), encephalopathy, cholangitis and mesenteric angina $[25,26,32,34,35]$. The etiology of ascites (whether derived from HOCF or portal hypertension) was established on the basis of clinical, laboratory and instrumental data.

\section{Laboratory Assessment}

At the time of patient enrollment, blood samples were taken for DNA extraction and mutational analysis. Measured at each checkup, we determined in each patient the following parameters: hemoglobin, serum iron, ferritin, AST, ALT, GGT, alkaline phosphatase, bilirubin, total protein, albumin, international normalized ratio (INR). Two parameters, INR and albumin, were used to evaluate synthetic liver functional efficiency. Abnormal values were expressed as the fold difference relative to the upper limit of normal $(\mathrm{n} \times \mathrm{ULN})$. Serum markers related to current or past hepatitis $\mathrm{C}$ and/or $\mathrm{B}$ virus infection were collected at the time of enrollment. Cirrhosis was diagnosed using internationally accepted criteria, including history, clinical/laboratory findings, and pertinent Doppler US findings [36].

\section{Instrumental Assessment}

As previously described, Doppler abdominal US scans were performed during enrollment and during follow-up visits [15, 37-39]. Based on the Doppler US findings, liver VMs were classified according to their severity grades as follows: minimal (0 to 1), moderate (2), severe (3), and decompensated (4) $[15,37,38]$. Patients were diagnosed with portal hypertension if the diameter of their portal vein was either $>13 \mathrm{~mm}$ or was associated with arterioportal shunts or splenomegaly (defined as craniocaudal diameter $>13 \mathrm{~cm}$ ).

Patients with liver VMs underwent echocardiographic examinations to evaluate cardiac function and morphology both at the time of enrollment and during each checkup. Using echocardiographic evaluation, we examined in each patient the following parameters: cardiac index $\left(1 / \mathrm{min} / \mathrm{m}^{2}\right)$, calculated as: cardiac output $\times\left[\left(\pi \times d^{2} / 4 \times \mathrm{VTI} \times\right.\right.$ cardiac frequency)/body surface area], where $d$ represents the 
diameter of the left ventricular outflow tract and VTI represents the sub-aortic velocity time integral. In cases where a patient suffered atrial fibrillation, the cardiac index was calculated using the average of at least ten cardiac cycles. Right ventricular systolic pulmonary arterial pressure $(\mathrm{mmHg})$, was calculated as: $[(4 \times$ the maximum velocity of tricuspid regurgitant jet $\left.{ }^{2}\right)+$ mean right atrial pressure].

An upper GI endoscopy was performed if either GI bleeding was suspected or if evaluation of portal hypertension patients was required, with further investigation [video capsule endoscopy (since 2001), colonoscopy] undertaken on the basis of pertinent findings.

When necessary, we performed additional tests that included the following: chest X-ray, computed tomography nuclear magnetic resonance imaging, liver scintigraphy (with $99 \mathrm{mTc}$ sulfur colloid), angiography, right heart catheterization, and portal pressure measurement (with a hepatic venous pressure gradient) [25].

\section{Follow-Up}

Depending on the severity of liver VMs found in a patient, we scheduled clinical and instrumental checkups for each patient. The amount of time between each was determined as follows: five years (for VM grades between 0 and 1), three years (for VM grades of 2), two years (for VM grades of 3), and between one month and one year (for VM grades of 4 and depending on the patient's specific clinical condition). To avoid losing patients, we called patients who missed their scheduled appointments to attempt to reschedule. Furthermore, patients could elect to schedule a follow-up for any reason that could potentially relate to HHT.

The total number of follow-up months to the last checkup was calculated for every patient.

\section{Treatment}

In patients with symptomatic liver involvement, we used multidisciplinary, clinical, biochemical, and instrumental approaches to correct the complications in a manner independent of the procedure for grading the liver VMs. Intense medical therapy was given to patients with HOCF NYHA class II to IV (including, as clinically needed, salt restrictions, diuretics, beta-blockers, digoxins, angiotensinconverting enzyme inhibitors, antiarrhythmic agents, cardioversions, and radiofrequency catheter ablation). The immediate goals of treating HOCF were to alleviate symptoms and to stabilize hemodynamic condition [40]. Treatment of portal hypertension complications (e.g., ascites or bleeding from gastroesophageal varices) and encephalopathy were undertaken as recommended in cirrhotic patients. Antibiotics were administered for cholangitis. Patients were also provided with supportive care, which included, when required, either blood transfusions or iron administration for purposes of both correcting anemia and treating of the source of bleeding (e.g., either epistaxis or gastrointestinal bleeding) in actively bleeding patients.

In patients who failed to respond to complication treatments and exhibited a progressive worsening of their clinical conditions, invasive treatments, which included transarterial embolization of liver VMs and orthotopic liver transplantation, were considered in order to eliminate hepatic VMs.

\section{Patient Outcomes}

The primary end-point of the present study was death related to a treated or untreated liver VM complication. There were two secondary end-points. The first was grouped as clinical parameters, i.e., the development of symptoms/signs of complicated liver VMs (HOCF complicated portal hypertension with ascites and/or gastrointestinal bleeding, encephalopathy, cholangitis and mesenteric angina, and, in general, any event related to either mesenteric or systemic circulation, because the liver has a unique hemodynamic relationship with both circulations) and the need for treatment of liver VMs. The second was biochemical, i.e., the development of cholestasis signs/symptoms. Patients with HHT but without liver VMs at initial screening were used as a control group to compare incidences of secondary end-points.

Response to therapy was defined as stable asymptomatic (where disappearance of symptoms/clinical signs of complicated liver VMs and, in particular, if HOCF returned to NYHA class I); stable but with ongoing symptoms (where improvement, but not disappearance, of clinical signs and symptoms and, in particular, if HOCF was downgraded to NYHA class III or II); or progressive (if worsening or death occurred despite treatment).

\section{Statistical Analysis}

Both cause-specific survival and time between the diagnosis of liver VMs and the development of symptoms or death were estimated using the Kaplan-Meier method.

Univariate analysis of continuous variables was performed using the Mann-Whitney rank sum test, and univariate analysis of categorical variables was performed using Fisher's exact test (two-tailed). Differences were considered statistically significant when $P<0.05$, and Bonferroni's correction for multiple comparisons was used for each end-point.

Statistical analyses were performed using SPSS (version 15; SPSS, Inc., Chicago, IL). 


\section{Results}

\section{Baseline Demographics}

Patient demographics (for both screened and included subjects) are shown in Fig. 1.

Baseline demographic, laboratory and radiological characteristics of the 154 HHT subjects with liver VMs are listed in Table 1. Altogether, 13 patients displayed symptoms during the initial examination (Tables 1 and 2).

The 154 patients were followed-up for a median period of 44 months (range 12-181), accounting for a total of 731 person-years.

During follow-up, the grading of liver VMs remained stable in 122 subjects (79\%) and worsened in 32 (21\%), with two subjects shifting from grade 0.5 to 1 and two subjects shifting to grade 2 from either grade 0.5 or grade 1 , seven subjects shifting to grade 2 to 3 , and ten shifting to grade 4 (three from grade 2 , seven from grade 3 ).

The 198 patients with HHT but without liver VMs formed our control group (Fig. 1). The 198 controls were followed-up for a median period of 42 months (range 11-179) accounting for a total of 836 person-years.

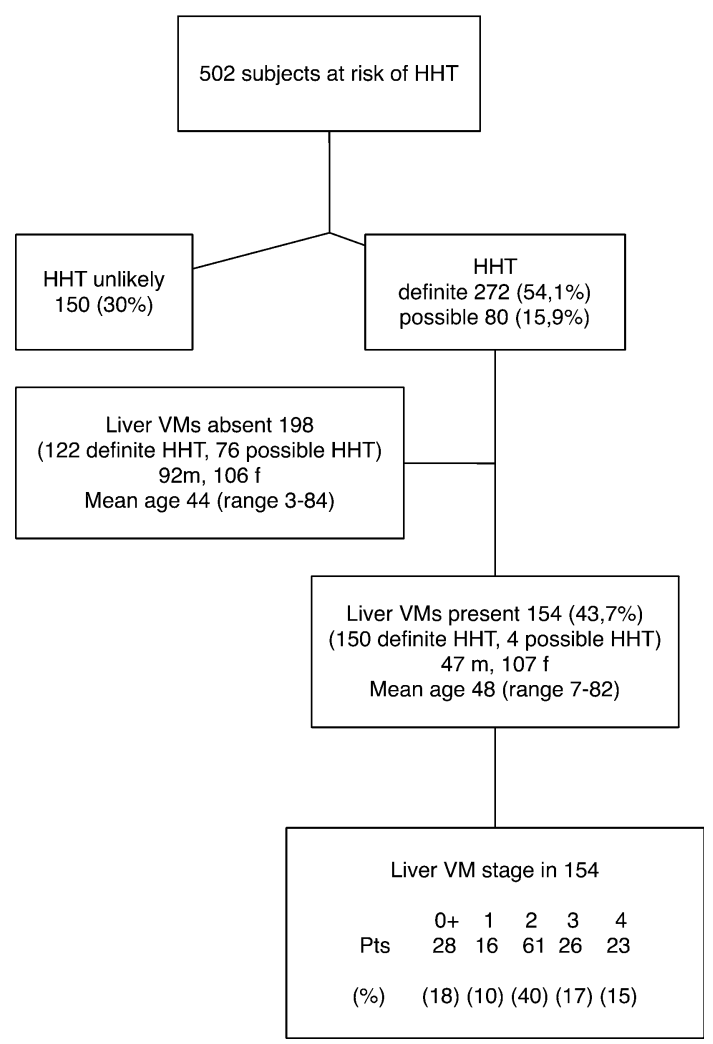

Fig. 1 Prevalence of hereditary hemorrhagic telangiectasia (HHT) and liver vascular malformations (VMs) in the study population
No subjects were lost to follow-up in both the patient group and the control group.

Clinical Course and Outcome

\section{Mortality}

Eight (5.2\%; seven females, one male, mean age at death 74, range 62-90) died of liver VM-related complications, as detailed in Table 2. The average incidence rate of fatal outcomes was 1.1 per 100 person-years (95\% confidence interval [CI] 0.3-1.8). The median survival time after diagnosis was 175 months (range 24-181 months, 95\% CI 66-283) in the Kaplan-Meier analysis (Fig. 2a).

There were two deaths in the 13 patients (15\%) who were symptomatic at the baseline evaluation (patients 4 and 5 in Table 2), and there were six deaths in the 141 patients (4\%) who were asymptomatic at baseline (patients 17, 20, 23, 28, 34, and 35 in Table 2); this difference was not statistically significant $(P=0.28)$.

All eight patients who died had stage 4 liver VMs at baseline (out of 23 patients who had stage 4 liver VMs; see Fig. 1), which was a significantly greater rate $(P<0.001)$ than in patients with other liver VMs stages between 0.5 and 3. Of the patients who died, one had HHT1, seven had HHT2, and seven had missense mutations.

Five deaths occurred in the same pedigree.

\section{Morbidity}

Of the 154 patients, 39 (25.3\%) had clinical events possibly related to liver VMs (Tables 2 and 3); after exclusion of $13(33 \%)$ subjects with symptoms related to VMs at baseline, the remaining 26 (67\%) developed symptoms during follow-up, with an incidence rate of 3.6 events per 100 person-years (95\% CI 2.2-4.9). The median event-free survival was 90 months (range 10-181, 95\% CI 44-135 months) (Fig. 2b).

The estimated median times to the development of HOCF, atrial fibrillation, portal hypertension, ascites and GI bleeding were 58 (range 15-172), 88 (range 2-151), 41 (range 16-136), 72 (range 27-177) and 23 (range 2-90) months, respectively.

The more common complications (listed as incidence rates per 100 person-years) were as follows: HOCF (1.4, 95\% CI 0.5-2.2); complicated portal hypertension (1.2, 95\% CI 0.4-1.9); and GI bleeding (1.0, 95\% CI 0.2-1.7).

In the control group, no patients had HOCF or portal hypertension (due to liver VMs), which was significantly less frequent than in the study population $(P=0.009)$. No control subject had ascites, encephalopathy, cholangitis, or mesenteric angina $(P=0.009$ for ascites; not significant for encephalopathy and mesenteric angina). 
Table 1 Baseline demographic, laboratory and instrumental imaging characteristics of 154 hereditary hemorrhagic telangiectasia (HHT) patients with liver vascular malformations (VMs)

\begin{tabular}{|c|c|c|c|c|c|c|}
\hline \multirow[t]{2}{*}{ Demographics } & \multicolumn{2}{|c|}{$\begin{array}{l}13 \text { patients symptomatic at } \\
\text { baseline }\end{array}$} & \multicolumn{2}{|c|}{$\begin{array}{l}141 \text { patients asymptomatic at } \\
\text { baseline }\end{array}$} & \multicolumn{2}{|l|}{ All 154 patients } \\
\hline & Mean $\pm \mathrm{SD}$ & Range & Mean $\pm \mathrm{SD}$ & Range & Mean $\pm \mathrm{SD}$ & Range \\
\hline Age $(y)$ & $51 \pm 16$ & $21-64$ & $47 \pm 15$ & $7-82$ & $48 \pm 16$ & $7-82$ \\
\hline Sex & 6 male, 7 female & & 41 male, 100 female & & 47 male, 107 female & \\
\hline \multicolumn{7}{|l|}{ Laboratory findings $^{\mathrm{a}}$} \\
\hline $\mathrm{Hb}, \mathrm{g} / \mathrm{dl}$ & $10.2 \pm 1.8$ & $7.0-13.1$ & $12.23 \pm 2.9$ & $5.6-19.2$ & $12.13 \pm 2.1$ & $5.6-19.2$ \\
\hline AST $(\mathrm{IU} / 1 \times \mathrm{ULN})$ & $1.1 \pm 0.3$ & $1-2$ & $1.0 \pm 0.3$ & $1-4$ & $1.1 \pm 0.3$ & $1-4$ \\
\hline $\operatorname{ALT}(\mathrm{IU} / 1 \times \mathrm{ULN})$ & $1.2 \pm 0.4$ & $1-2$ & $1.37 \pm 0.4$ & $1-4$ & $1.3 \pm 0.4$ & $1-4$ \\
\hline GGT $(\mathrm{IU} / 1 \times \mathrm{ULN})$ & $3.46 \pm 3.1$ & $1-10$ & $1.32 \pm 1.2$ & $1-8$ & $1.52 \pm 1.2$ & $1-10$ \\
\hline Alk phosphatase $(\mathrm{IU} / 1 \times \mathrm{ULN})$ & $1.28 \pm 0.4$ & $1-2$ & $1.0 \pm 0.2$ & $1-3$ & $1.08 \pm 0.2$ & $1-3$ \\
\hline Bilirubin $(\mathrm{mg} / \mathrm{dl} ; \times \mathrm{ULN})$ & $1.15 \pm 0.5$ & $1-3$ & $1.0 \pm 0.1$ & $1-3$ & $1.02 \pm 0.1$ & $1-3$ \\
\hline INR & $1.03 \pm 0.07$ & $1-1.2$ & $1.0 \pm 0.1$ & $1-2$ & $1.03 \pm 0.1$ & $1-2$ \\
\hline Albumin $(\mathrm{g} / \mathrm{dl})$ & $3.98 \pm 0.1$ & $3.8-4.2$ & $4.33 \pm 0.2$ & $2.9-4.8$ & $4.30 \pm 0.2$ & $2.9-4.8$ \\
\hline \multicolumn{7}{|l|}{ Doppler US and echocardiography ${ }^{\mathrm{b}}$} \\
\hline \multicolumn{7}{|l|}{ Hepatic artery } \\
\hline Inner diameter $(\mathrm{mm})$ & $11.3 \pm 4.5$ & $6.3-22$ & $7.78 \pm 3.2$ & $5.1-17$ & $8.18 \pm 2.7$ & $5.1-22$ \\
\hline Resistance index & $0.53 \pm 0.08$ & $0.40-0.68$ & $0.55 \pm 0.1$ & $0.34-0.75$ & $0.54 \pm 0.1$ & $0.34-0.75$ \\
\hline Peak flow velocity $(\mathrm{cm} / \mathrm{sec})$ & $127.6 \pm 35.1$ & $77-210$ & $106.0 \pm 25.4$ & $75-212$ & 108.0425 .4 & $75-212$ \\
\hline \multicolumn{7}{|l|}{ Portal vein } \\
\hline Inner diameter $(\mathrm{mm})$ & $11.4 \pm 3.0$ & $8-16$ & $8.78 \pm 2.7$ & $6-21$ & $8.9 \pm 2.7$ & $6-21$ \\
\hline Mean flow velocity $(\mathrm{cm} / \mathrm{sec})$ & $17.0 \pm 5.0$ & $11-26$ & $19.45 \pm 4.6$ & $8-33$ & $18.90 \pm 3.6$ & $8-33$ \\
\hline \multicolumn{7}{|l|}{ Hepatic vein } \\
\hline Inner diameter $(\mathrm{mm})$ & $10.3 \pm 3.7$ & $7-18$ & $7.89 \pm 2.2$ & $5-17$ & $8.16 \pm 2.2$ & $5-18$ \\
\hline Peak flow velocity $(\mathrm{cm} / \mathrm{sec})$ & $37.0 \pm 11.5$ & $22-57$ & $34.7 \pm 7.4$ & $20-76$ & $35.2 \pm 8.4$ & $20-76$ \\
\hline \multicolumn{7}{|l|}{ Focal nodular hyperplasia } \\
\hline Number & $0.38 \pm 0.6$ & $0-2$ & $0.15 \pm 0.7$ & $1-5$ & $0.23 \pm 0.6$ & $0-5$ \\
\hline Diameter (cm) & $1.0 \pm 1.7$ & $0-5.2$ & $0.76 \pm 1.9$ & $2.2-20.7$ & $0.79 \pm 2.5$ & $0-20.7$ \\
\hline Cardiac Index $\left(1 / \mathrm{min} / \mathrm{m}^{2}\right)^{\mathrm{c}}$ & $4.3 \pm 1.0$ & $2.9-10.7$ & 3. $2 \pm 0.2$ & $2.7-10.1$ & $3.3 \pm 0.4$ & $2.7-10.7$ \\
\hline Pulmonary artery pressure $(\mathrm{mmHg})$ & $34.2 \pm 9.2$ & $24-50$ & $27.10 \pm 4.0$ & $21-48$ & $28.14 \pm 6.0$ & $21-50$ \\
\hline
\end{tabular}

$H b$ hemoglobin, $G G T$ gammaglutamyltranspeptidase

${ }^{a}$ Eight patients were anti-HCV positive (5/8 HCV RNA positive); two patients were HBsAg positive and HBV DNA negative

b Nodularity of the liver surface was found on Doppler US in five patients; focal or diffuse dilatation of intrahepatic bile ducts was found in two patients

c Measured in 145/154 at baseline

\section{HOCF}

A potential precipitating factor for $\mathrm{HOCF}$ was found in eight of 14 patients: anemia was found in seven patients and evidence of steroid treatment was found in one.

\section{Atrial Fibrillation}

Atrial fibrillation occurred in $12 / 154$ patients with hepatic VMs (1.6 incidence rate per 100 person-years, 95\% CI 0.6-2.5). Two out of 198 controls had atrial fibrillation, for an incidence rate of $0.2(95 \%$ CI $0.1-0.5)(P<0.001)$.
Of nine patients with paroxysmal atrial fibrillation, eight had a mean of six recurrences (range 2-15), and seven of these eight patients developed chronic atrial fibrillation after a mean interval of 36 months (range 2-80). Two with paroxysmal atrial fibrillation did not develop HOCF after 16 and 74 months of follow-up, respectively.

Of the ten patients with chronic atrial fibrillation, arrhythmia preceded HOCF in six patients by a mean interval of 45 months (range 6-88); in one patient, the onsets of both arrhythmia and HOCF occurred simultaneously; in two, it followed HOCF by 38 and 59 months, respectively; in one, $\mathrm{HOCF}$ had not developed at the 95-month follow-up. 
Table 2 Clinical events in 39/154 patients with hereditary hemorrhagic telangiectasia (HHT) and liver vascular malformations (VMs)

\begin{tabular}{|c|c|c|c|c|c|c|c|c|c|c|c|c|}
\hline Patient & $\operatorname{Age}^{a}(y)$ & Sex & $\begin{array}{l}\text { Liver } \\
\text { VMs } \\
\text { stage }^{\text {a }}\end{array}$ & $\mathrm{HOCF}$ & $\begin{array}{l}\text { Atrial } \\
\text { fibrillation }\end{array}$ & $\begin{array}{l}\text { Supraventricular } \\
\text { tachycardia }\end{array}$ & $\begin{array}{l}\text { Portal } \\
\text { hypert }\end{array}$ & Ascites & Encephalopathy & $\begin{array}{l}\text { GI } \\
\text { bleeding }\end{array}$ & $\begin{array}{l}\text { Abdominal } \\
\text { angina }\end{array}$ & Outcome \\
\hline 1 & 37 & M & 4 & & & 1 & 1 & & & 1 & $1^{\mathrm{b}}$ & Alive \\
\hline 2 & 62 & $\mathrm{~F}$ & 2 & & & & & & & $1^{\mathrm{b}}$ & & Alive \\
\hline 3 & 79 & M & 4 & $1^{\mathrm{b}}$ & 1 & & & 1 & & & & Alive \\
\hline 4 & 61 & $\mathrm{~F}$ & 4 & $1^{\mathrm{b}}$ & 1 & & & 1 & & & & Dead \\
\hline 5 & 64 & M & 4 & & & & 1 & & & $1^{\mathrm{b}}$ & & Dead \\
\hline 6 & 79 & M & 4 & & & & 1 & & & $1^{\mathrm{b}}$ & & Alive \\
\hline 7 & 49 & $\mathrm{~F}$ & 4 & & & & 1 & & & $1^{\mathrm{b}}$ & & Alive \\
\hline 8 & 64 & $\mathrm{~F}$ & 4 & $1^{\mathrm{b}}$ & & & & & & & & Alive \\
\hline 9 & 57 & $\mathrm{~F}$ & 4 & $1^{\mathrm{b}}$ & 1 & & & & & & & Alive \\
\hline 10 & 56 & $\mathrm{~F}$ & 2 & & & & & & & $1^{\mathrm{b}}$ & & Alive \\
\hline 11 & 39 & $\mathrm{~F}$ & 4 & & & & 1 & 1 & & & & Alive \\
\hline 12 & 64 & M & 2 & & & & & & & $1^{\mathrm{b}}$ & & Alive \\
\hline 13 & 62 & M & 1 & & & & & & & $1^{\mathrm{b}}$ & & Alive \\
\hline 14 & 73 & M & 3 & $1^{\mathrm{b}}$ & & & & 1 & & & & Alive \\
\hline 15 & 22 & $\mathrm{~F}$ & 4 & $1^{\mathrm{b}}$ & 1 & & 1 & & & & & Alive \\
\hline 16 & 69 & $\mathrm{~F}$ & 4 & $1^{\mathrm{b}}$ & & & & & & & & Alive \\
\hline 17 & 48 & $\mathrm{~F}$ & 4 & & & & 1 & $1^{\mathrm{b}}$ & & & & Dead \\
\hline 18 & 48 & $\mathrm{~F}$ & 4 & $1^{\mathrm{b}}$ & 1 & & 1 & 1 & & 1 & & Alive \\
\hline 19 & 36 & $\mathrm{~F}$ & 4 & $1^{\mathrm{b}}$ & 1 & & & 1 & & & & Alive \\
\hline 20 & 59 & $\mathrm{~F}$ & 4 & $1^{\mathrm{b}}$ & 1 & & & 1 & & & & Dead \\
\hline 21 & 69 & $\mathrm{~F}$ & 3 & & & $1^{\mathrm{b}}$ & & & & & & Alive \\
\hline 22 & 69 & $\mathrm{~F}$ & 2 & & & & & & $1^{\mathrm{b}}$ & & & Alive \\
\hline 23 & 74 & $\mathrm{~F}$ & 4 & & & & 1 & $1^{\mathrm{b}}$ & & & & Dead \\
\hline 24 & 64 & M & 2 & & & & 1 & 1 & & $1^{\mathrm{b}}$ & & Alive \\
\hline 25 & 31 & M & 3 & $1^{\mathrm{b}}$ & & & & 1 & & & & Alive \\
\hline 26 & 69 & $\mathrm{~F}$ & 4 & & & & 1 & & 1 & $1^{\mathrm{b}}$ & & Alive \\
\hline 27 & 70 & $\mathrm{~F}$ & 4 & & $1^{\mathrm{b}}$ & & 1 & & & & & Alive \\
\hline 28 & 64 & $\mathrm{~F}$ & 4 & $1^{\mathrm{b}}$ & & & 1 & 1 & & & & Dead \\
\hline 29 & 38 & M & 3 & & $1^{\mathrm{b}}$ & & & & & & & \\
\hline 30 & 57 & M & 2 & & & & & & & $1^{\mathrm{b}}$ & & Alive \\
\hline 31 & 49 & M & 2 & & & & & & & $1^{\mathrm{b}}$ & & Alive \\
\hline 32 & 52 & $\mathrm{~F}$ & 2 & & $1^{\mathrm{b}}$ & & & & & & & Alive \\
\hline 33 & 58 & M & 3 & $1^{\mathrm{b}}$ & 1 & & & & & & & Alive \\
\hline 34 & 82 & $\mathrm{~F}$ & 4 & $1^{\mathrm{b}}$ & 1 & & & & & & & Dead \\
\hline 35 & 82 & $\mathrm{~F}$ & 4 & & & & 1 & & & $1^{\mathrm{b}}$ & & Dead \\
\hline 36 & 56 & M & 3 & & & & 1 & $1^{\mathrm{b}}$ & & & & Alive \\
\hline 37 & 62 & $\mathrm{~F}$ & 3 & & & & 1 & & & $1^{\mathrm{b}}$ & & Alive \\
\hline 38 & 52 & $\mathrm{~F}$ & 3 & & & $1^{\mathrm{b}}$ & & & & & & Alive \\
\hline 39 & 39 & $\mathrm{~F}$ & 2 & & & $1^{\mathrm{b}}$ & & & & & & Alive \\
\hline Total $^{\mathrm{c}}$ & & & & $14(9)$ & $12(8)$ & $4(3)$ & $16(10)$ & $13(8)$ & $2(1)$ & $15(10)$ & $1(1)$ & Alive \\
\hline
\end{tabular}

HOCF high-output cardiac failure

The first 13 patients had symptoms at enrolment. Patient 37 had coexistent HCV-related compensated cirrhosis. Patient 18 had mild arterial hypertension

a At diagnosis

b The clinically dominant feature, and which was the death cause in cases with adverse outcome

${ }^{c}$ Percentages in brackets (number of complications/154 pts) are rounded to the next integer for decimal points $>0.5$ 
Fig. 2 Overall survival (a) and event-free survival (b) curves of 154 hereditary hemorrhagic telangiectasia (HHT) subjects with liver vascular malformations (VMs)
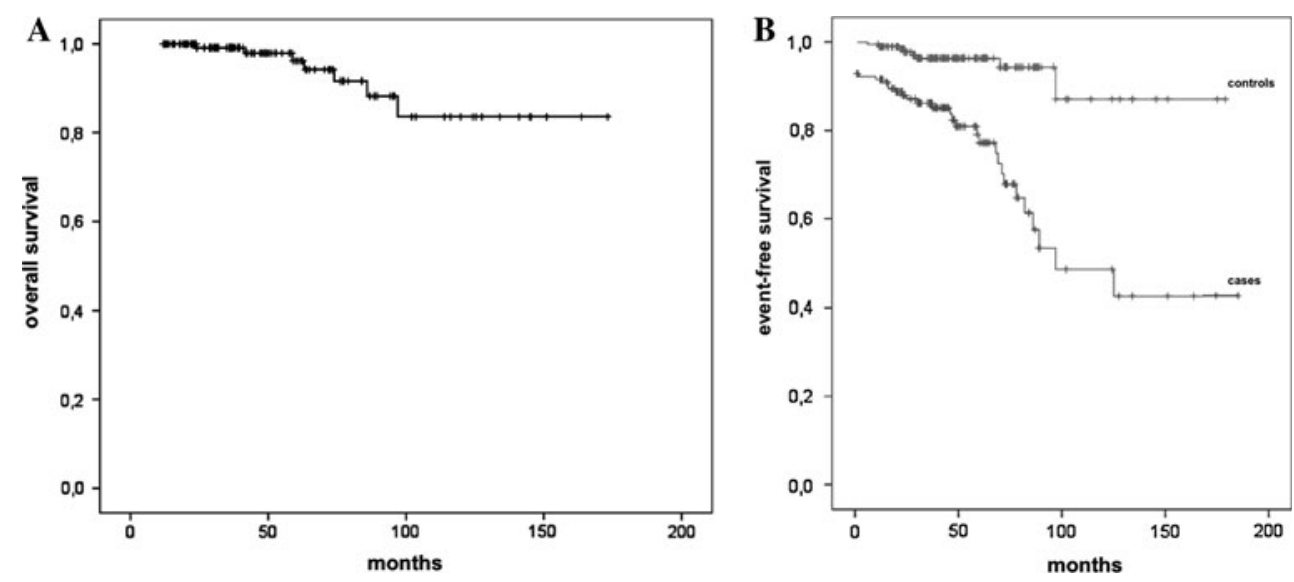

Table 3 Analysis of clinical events by liver vascular malformations (VM) stage

\begin{tabular}{|c|c|c|c|c|c|c|c|c|c|}
\hline $\begin{array}{l}\text { Liver VMs } \\
\text { stage }^{\mathrm{a}}\end{array}$ & $\mathrm{HOCF}$ & $\begin{array}{l}\text { Atrial } \\
\text { fibrillation }\end{array}$ & $\begin{array}{l}\text { Supraventricular } \\
\text { tachycardia }\end{array}$ & $\begin{array}{l}\text { Portal } \\
\text { hypert }+ \\
\text { ascites }\end{array}$ & $\begin{array}{l}\text { Portal } \\
\text { hypertension }+ \\
\text { GI bleeding }\end{array}$ & Encephalopathy & $\begin{array}{l}\text { GI } \\
\text { bleeding }\end{array}$ & $\begin{array}{l}\text { Abdominal } \\
\text { angina }\end{array}$ & Total \\
\hline $0+$ & 0 & 0 & 0 & 0 & 0 & 0 & 0 & 0 & 0 \\
\hline 1 & 0 & 0 & 0 & 0 & 0 & 0 & 1 & 0 & 1 \\
\hline 2 & 0 & 1 & 1 & 1 & 1 & 1 & 5 & 0 & 10 \\
\hline 3 & 3 & 2 & 2 & 1 & 1 & 0 & 0 & 0 & 9 \\
\hline 4 & 11 & 9 & 1 & 5 & 7 & 1 & 0 & 1 & 35 \\
\hline Total & 14 & 12 & 4 & 7 & 9 & 2 & 6 & 1 & 55 \\
\hline
\end{tabular}

HOCF high-output cardiac failure

a At baseline

Supraventricular tachycardia occurred in four patients (mean number of episodes was 6; range 2-15) and was not associated with HOCF in any case. No control had supraventricular tachycardia $(P=0.36)$.

\section{GI Bleeding}

GI bleeding was due in all cases to upper GI telangiectasias. Six of 198 (3\%, with an incidence rate per 100 personyears of $0.7,95 \%$ CI 5.2-8.7) controls had GI bleeding; the difference compared to the study population was not significant $(P=0.36)$.

\section{Anemia}

The 39 patients who had complications had a mean value of hemoglobin at baseline of 11.4 (range 5.6-15.9) and of 7.1 (range 3.4-11.3) at the last checkup; in the 115 patients not exhibiting complications, the mean values of hemoglobin at baseline were 12.12 (range 5.6-19.2) and 11.8 (range 5.4-16.6) at the last checkup. Differences between the two groups of patients were not statistically significant.

\section{Genotype}

Genotype (available for 110 out of 154 HHT subjects with liver VMs) showed an ENG (HHT1) mutation in 27 cases (18\%) and an ACVRL1(HHT2) mutation in 83 cases (54\%). Mutational analysis (available for 99 patients) revealed a truncating mutation in 33 patients $(21 \%)$, a missense mutation in 44 patients $(28 \%)$, an in-frame deletion in 16 patients $(10 \%)$, and a splicing mutation in six patients $(3 \%)$.

\section{Estroprogestinics}

Estroprogestinics were taken before liver VMs diagnosis by 28 women for a mean of 32.3 months (range 1-96). During follow-up, 14 women were found to have a mean estroprogestinic intake of 28.7 months (range 1-74).

\section{Pregnancies}

A mean of 2.07 (range 1-9) pregnancies was recorded in 75 women before diagnosis. During follow-up, three patients had two pregnancies each. Symptoms related to 
complications involving liver VMs were not reported in any of the 155 at-term pregnancies.

\section{Response to Treatments}

Appropriate treatment was intensively administered to every patient showing liver VM-related complications. In four patients (non-responders to previous intensive treatments), transarterial embolization (in two) and liver transplant (in two) were performed.

\section{HOCF}

Salt restriction and diuretics, beta-blockers, digoxin, and angiotensin-converting enzyme inhibitors were used to treat 14 cases of HOCF. Nine patients became stably asymptomatic; there was no response, with progression to death, in two. Staged HA embolization was attempted in two cases of HOCF who were not responsive to previous treatments and rejected from liver transplantation, with a transient response in one case but eventual progression to death in both.

Liver transplantation was performed in one patient with HOCF who was not responsive to previous treatments. In this patient, cardiac and pulmonary hemodynamic assessments (echocardiography, right heart catheterization) showed both right and left heart severe dilatation, a cardiac index of $6.2 \mathrm{l} / \mathrm{min} / \mathrm{m}^{2}$, and pulmonary hypertension with a pulmonary arterial systolic pressure of $55 \mathrm{mmHg}$. These data, which had shown a progressive worsening over 1 year in spite of intensive medical treatment, together with the lack of clinical response and persistence of cardiac failure NYHA class III-IV, led to the decision of liver transplantation. After 14 months following a successful transplantation, the patient showed continued stability and was symptom-free.

\section{Arrhythmias}

To treat atrial fibrillation, antiarrhythmic agents (e.g., amiodarone and flecainide), calcium channel blockers, beta-blockers, digoxin or electrical cardioversion were used.

Conversion to sinus rhythm was obtained in two patients (16\%) without HOCF. Partial response, as shown by slowing of the ventricular rate, was found in the remaining $10 / 12$ patients, including the three patients in which atrial fibrillation presented with or after HOCF. Conversion to sinus rhythm was obtained in the $2 / 198$ controls who presented with atrial fibrillation during follow-up.

To treat supraventricular tachycardia in four patients, antiarrhythmic agents, beta-blockers, amiodarone, and flecainide were used. The treatment yielded a complete response in three cases; one patient not responsive to antiarrhythmics was successfully treated with RF ablation.

\section{Complicated Portal Hypertension}

Salt restriction and diuretics were used to treat ascites associated with portal hypertension, with complete response in four cases and lack of response and progression to death in two cases. Liver transplantation was performed in another case where the patient had refractory ascites, and it proved successful, as the patient remained stable and asymptomatic through the 56-month follow-up.

In six additional patients, GI bleeding was treated with tranexamic acid, danazol, octreotide, and endoscopic treatment with argon plasma coagulation. All patients progressed to a stable asymptomatic condition.

\section{GI Bleeding}

In nine patients with GI bleeding associated with portal hypertension, beta-blockers/octreotide, endoscopic treatment with argon plasma coagulation of telangiectases, or surgery (gastrectomy) were used. After treatment, six patients were stable and asymptomatic, one was stable but with ongoing symptoms, and two eventually died.

\section{Abdominal Angina}

One patient was treated with analgesics, and the patient became stable but exhibited ongoing symptoms. HA-staged embolization was then attempted, which similarly yielded a stable patient with ongoing symptoms.

\section{Encephalopathy}

Encephalopathy was successfully treated in two patients with lactulose and antibiotics.

\section{Outcome of Treatments}

The outcome of treatments in 55 complications observed in 39 patients (Table 3) included: complete response with stable condition in $35(63.7 \%)$, partial response with ongoing symptoms in $12(21.8 \%)$, and no response (with progression to death) in eight (14.5\%).

Among the 15 complications occurring in the 13 patients who were symptomatic at baseline, treatments yielded five complete responses (33\%), compared with 30 complete responses $(75 \%)$ in 40 complications occurring in 26 patients who were asymptomatic at baseline. Response to 
treatment in this latter group proved significantly better $(P=0.01)$.

Changes in liver VM grading after treatment were not observed in any of the treated patients.

\section{Discussion}

To date, data concerning the natural history of liver involvement in HHT, derived from a longitudinal cohort study, are lacking. However, such data could clearly have a substantial impact on the clinical management of HHT. The key finding of this large cohort study is to provide the first clear-cut evidence regarding the progression and outcome of liver VMs in HHT patients, with event rates of 3.6 and 1.1 per 100 person-years, respectively.

The strengths of the study include (1) the potential to confirm that HOCF represents the predominant complication associated with HHT and that HOCF accounts for half of hepatic VM-associated fatalities [41]; (2) arrhythmias emerged as an important complication of hepatic VMs; (3) our finding that all patients who died had stage 4 liver VMs at baseline, which was a significantly higher rate when compared with patients with other liver VM stages, clearly defines a patient subgroup at high risk for poor outcome, who need the most intensive clinical management; (4) our study allowed us to evaluate patient's response to treatment. With respect to patient response to therapy for liver complications, $63 \%$ of patients had a complete response, and $21 \%$ of patients had a partial response; these high response rates demonstrate that caution should be exercised when considering major treatment methods, particularly embolization, which is not only risky but also palliative [25, 32]; and (5) the 90 month interval between the diagnosis and the onset of symptoms observed in the present cohort is the time span in which potentially effective preventive measures could prevent or slow the course of the disease.

A limitation of the present study, as is usual in natural history studies, is that recruitment consecutively included either asymptomatic or symptomatic subjects and who were likely to fall in different disease stages. To estimate the lead-time bias in the interpretation of survival results, we also provided the absolute mortality rates in the patients who were symptomatic or asymptomatic at the diagnosis. The lack of statistical significance in the difference between the absolute mortality rates in the symptomatic patients compared to the absolute mortality rates of asymptomatic patients at diagnosis (15 vs. $4 \%$ ) actually translates to a non significant lead-time bias in the present study.

Previous studies regarding liver VMs in HHT consist of anecdotal reports, cross-sectional studies $[15,16,28,37$, 38 ] dealing with the prevalence of hepatic involvement in
HHT, case series including only symptomatic patients [26], and expert reviews [23, 27, 41].

In our study cohort, we found 154 patients with HHT and liver VMs by systematically screening subjects at risk for HHT; therefore, our participants provide an adequate representation of the entire spectrum of patients who have liver VMs. This is confirmed by the distribution of liver VMs stage shown in Fig. 1. The epidemiological scenario depicted by our study shows marked differences from what was previously hypothesized on the basis of prior reports, though these reports were not dealing with natural history $[23,26,27]$.

Because this study included patients with chronic cardiac overload due to liver VMs, we considered noteworthy every cardiac event, including isolated cardiac arrhythmias. In fact, the significant difference in arrhythmia rates between subjects with liver VMs and control subjects supports the hypothesis that arrhythmias in patients with liver VMs are not purely coincidental and should be approached with special caution.

In contrast to previous studies, complicated portal hypertension occurred at a rate comparable to that of HOCF, accounting for the other half of HHT-associated fatalities. A reasonable hypothesis to explain the increased incidence of complicated portal hypertensionis is that in the past, this complication was misdiagnosed as cirrhosis [41]. We therefore recommend a close follow-up with timely medical and/or endoscopic treatment of complications.

As previously noted in a case series [26], the hemorrhagic complications were not due to variceal bleeding but were due rather to bleeding from GI telangiectasias. In our study, GI bleeding events were collected even in the absence of portal hypertension; these events were used as covariates in the event-free survival analysis. The difference in GI bleeding rates between subjects with liver VMs and control subjects (10 vs. 3\%), even though it did not reach statistical significance, suggests that a link exists between GI bleeding and liver VMs. Probably, when this complication is associated with portal hypertension, mesenteric congestion due to portal hypertension triggers and tends to maintain bleeding from GI telangiectasias, as suggested by the fact that nine of our 15 patients who experienced GI bleeding had portal hypertension.

An ominous complication associated with hepatic VMs is bile duct ischemia, which can lead to cholangitis and bile duct necrosis, which may result in the need for emergent orthotopic liver transplantation [25, 42-44]. Notably, despite the presence of anicteric cholestasis in one-third of our patients, we detected no cases of cholangitis. Clinical awareness of the presence and severity of liver VMs, as determined by screenings, subsequent follow-ups, and timely therapeutic interventions, may have helped reduce the rate of this complication. 
In contrast to previous studies, we observed few cases of abdominal angina and encephalopathy [23, 25, 41]. Finally, we observed no adverse events related to liver VMs during pregnancy .

Our data support the use of the follow-up schedule used in the present study, with greater checkup frequency indicated for patients with liver VM stage 4. Moreover, these data suggest that Doppler US not only can accurately diagnose and stage liver VMs [15, 28, 33, 38, 45, 46], but also can provide prognostic indications of liver VMs. These data further support the clinical utility of using this technique to screen for liver VMs [25, 32].

The causes of treatment failures in the present series are likely to be multifactorial (e.g., anemia and atrial dilatation). To explain the treatment failure rate $(14 \%)$, we speculate that most of the patients live a far distance from our center, which is a tertiary HHT referral center that attracts patients from all over Italy, making it more difficult to supervise the clinical management of patients. The difficulty of supervising patients underscores the importance of providing detailed instructions to local physicians caring for HHT patients outside of the tertiary care referral center to allow them to deal with both current and potential complications in a timely manner.

The longitudinal series highlights a problem that is commonly encountered in HHT patients with complicated liver VMs (i.e., the treatment of atrial fibrillation). Atrial fibrillation, which in the present series was associated with HOCF in nine cases and heralded HOCF in six, when occurring in the context of HHT with chronic cardiac overload due to liver VMs, requires maximum effort to obtain rhythm control, either via cardioversion or via antiarrhythmic drugs, because arrhythmia can critically reduce cardiac hemodynamic efficiency. However, it should be noted that in these patients, substantial left atrial enlargement is common, which reduces the success rate of antiarrhythmic therapy. Moreover, in HHT patients, anticoagulation also presents difficulties because it can cause bleeding increase due to telangiectasias. Therefore, in this context, the heart rate control can be realistically considered a success because there is no clear evidence that restoring sinus rhythm is superior to controlling rate in reducing morbidity and mortality in patients with persistent atrial fibrillation and heart failure [40]. The clinical importance of HOCF also highlights the importance of treating conditions that can precipitate cardiac failure. Thus, both aggressive correction of a patient's anemia and maximum efforts to treat a patient's bleeding source should be given to patients with predictors of severe outcome of liver VMs. Additionally, in these patients, drugs that could precipitate volume overload, such as steroids or IV fluids, should be avoided.
The data of our study do not present strong arguments for screening asymptomatic individuals with HHT, because we did not treat patients prior to the onset of symptoms, and because all treatments performed in symptomatic patients did not alter hepatic VM grade. However, the significantly better outcome seen in treatments given to the subgroup of patients who were asymptomatic at baseline, where hepatic VMs were diagnosed by Doppler US screening, seems to provide a favorable argument for screening for liver VMs. Altogether, identifying patient conditions by the initial screening enables the early identification of liver VM complications, and in particular of cardiac decompensation, which is a key point for treatment success [40]. Advantages provided by the prior recognition of the condition have been similarly found in the case of pulmonary VMs in HHT [30].

Past studies show the potential for preventive treatments for hepatic VM complications regardless of whether HHT is present. These studies suggest that, for cardiac overload, either angiotensin-converting enzyme inhibitors or carvedilol could be used to prevent cardiac remodeling; for portal hypertension, beta-blockers could prevent GI bleeding from varices or from GI telangiectasias [47]. Antiangiogenic drugs, such as bevacizumab, which has induced a reduction in liver VMs in a few anecdotal reports, should be studied in controlled trials that involve patients who exhibit predictors of a poor outcome [48, 49].

In conclusion, our study shows that the progression of hepatic VMs occurs in a considerable number of cases. Our study identifies a patient subgroup at high risk for a poor outcome; patients in this subgroup require the most intensive forms of clinical management and surveillance.

Acknowledgments The study was funded by the Italian Hereditary Hemorrhagic Telangiectasia (HHT) Foundation "Onilde Carini" with a grant donated by Dr. Eda Berger Vidale, whose contribution the authors gratefully and wholeheartedly acknowledge.

Conflict of interest The authors declare that they do not have anything to disclose regarding funding from industries or conflict of interest with respect to this manuscript.

Open Access This article is distributed under the terms of the Creative Commons Attribution Noncommercial License which permits any noncommercial use, distribution, and reproduction in any medium, provided the original author(s) and source are credited.

\section{References}

1. Guttmacher AE, Marchuk DA, White RI. Hereditary hemorrhagic telangiectasia. NEJM. 1995;333:918-924.

2. Dakeishi M, Shioya T, Wada Y, et al. Genetic epidemiology of hereditary hemorrhagic telangiectasia in a local community in the northern part of Japan. Hum Mutat. 2002;19:140-148. 
3. Govani FS, Shovlin CL. Hereditary hemorrhagic telangiectasia: a clinical and scientific review. Eur J Hum Gen. 2009;17:860-871.

4. Abdalla SA, Letarte M. Hereditary hemorrhagic telangiectasia: current views on genetics and mechanism of disease. J Med Genet. 2006;43:97-110.

5. Kjeldsen AD, Vase P, Green A. Hereditary haemorrhagic telangiectasia: a population-based study of prevalence and mortality in Danish patients. J Intern Med. 1999;245:31-39.

6. Bideau A, Brunet G, Heyer E, et al. An abnormal concentration of cases of Rendu-Osler disease in the Valserine valley of the French Jura: a genealogical and demographic study. Ann Hum Biol. 1992;19:233-247.

7. Shovlin CL, Guttmacher AE, Buscarini E, et al. Diagnostic criteria for hereditary hemorrhagic telangiectasia (Osler-WeberRendu syndrome). Am J Med Genet. 2000;91:66-67.

8. McDonald MT, Papenberg KA, Ghosh S, et al. A disease locus for hereditary haemorrhagic telangiectasia maps to chromosome 9q33-34. Nat Genet. 1994;6:197-204.

9. Shovlin C, Hughes JMB, Tuddenham EGD, et al. A gene for hereditary hemorrhagic telangiectasia maps to chromosome $9 \mathrm{q} 3$. Nat Genet. 1994;6:205-209.

10. Vincent P, Plauchu H, Hazan J, et al. A third locus for hereditary hemorrhagic telangiectasia maps to chromosome 12q. Hum Mol Genet. 1995;4:945-949.

11. Johnson DW, Berg JN, Baldwin MA, et al. Mutations in the activin receptor-like kinase 1 gene in hereditary hemorrhagic telangiectasia type 2. Nat Genet. 1996;13:189-195.

12. Gallione CJ, Repetto GM, Legius E, et al. A combined syndrome of juvenile polyposis and hereditary haemorrhagic telangiectasia associated with mutations in MADH4 (SMAD4). Lancet. 2004;363: 852-859.

13. Cole SG, Begbie ME, Wallace GM. Shovlin CLA new locus for hereditary haemorrhagic telangiectasia (HHT3) maps to chromosome 5. J Med Genet. 2005;42:577-582.

14. Bayrak-Toydemir P, McDonald J, Akarsu N, et al. A fourth locus for hereditary hemorrhagic telangiectasia maps to chromosome 7. Am J Med Genet. 2006;140:2155-2162.

15. Buscarini E, Danesino C, Olivieri C, et al. Doppler ultrasonographic grading of hepatic vascular malformations in hereditary hemorrhagic telangiectasia: results of extensive screening. Ultraschall Med. 2004;25:348-355.

16. Stabile Ianora AA, Memeo M, Sabbà C, et al. Hereditary hemorrhagic telangiectasia: multi-detector row helical CT assessment of hepatic involvement. Radiology. 2004;230:350-359.

17. Bayrak-Toydemir P, McDonald J, Markewitz B, et al. Genotypephenotype correlation in hereditary hemorrhagic telangiectasia: mutations and manifestations. Am J Med Genet A. 2006;140: 463-470.

18. Lesca G, Olivieri C, Burnichon N, et al. Genotype-phenotype correlations in hereditary hemorrhagic telangiectasia: data from the French-Italian HHT network. Genet Med. 2007;9:14-22.

19. Sabbà C, Pasculli G, Lenato GM, et al. Hereditary hemorrhagic telangiectasia: clinical features in ENG and ALK1 mutation carriers. J Thromb Haemost. 2007;5:1149-1157.

20. Letteboer TG, Mager JJ, Snijder RJ, et al. Genotype-phenotype relationship in hereditary haemorrhagic telangiectasia. $J$ Med Genet. 2006;43:371-377.

21. Brakensiek K, Frye-Boukhriss H, Mälzer M, et al. Detection of a significant association between mutations in the ACVRL1 gene and hepatic involvement in German patients with hereditary haemorrhagic telangiectasia. Clin Genet. 2008;74:171-177.

22. Fernandez-L A, Sanz-Rodriguez F, Zarrabeitia R, et al. Blood outgrowth endothelial cells from hereditary haemorrhagic telangiectasia patients reveal abnormalities compatible with vascular lesions. Cardiovasc Res. 2005;68:235-248.
23. Buscarini E, Danesino C, Olivieri C, et al. Liver involvement in hereditary haemorrhagic telangiectasia or Rendu-Osler-Weber disease. Dig Liver Dis. 2005;37:635-645.

24. Shovlin CL, Winstock AR, Peters AM, et al. Medical complications of pregnancy in hereditary haemorrhagic telangiectasia. QJM. 1995;88:879-887.

25. Buscarini E, Plauchu H, GarciaTsao G, et al. Liver involvement in hereditary hemorrhagic telangiectasia: consensus recommendations. Liver Int. 2006;26:1040-1046.

26. Garcia-Tsao G, Korzenik J, Young L, et al. Liver disease in patients with hereditary hemorrhagic telangiectasia. NEJM. 2000;343: 931-936.

27. Khalid SK, Garcia-Tsao G. Hepatic vascular malformations in hereditary hemorrhagic telangiectasia. Semin Liver Dis. 2008;28: 247-258.

28. Gincul R, Lesca G, Gelas-Dore B, et al. Evaluation of previously non screened hereditary hemorrhagic telangiectasia patients shows frequent liver involvement and early cardiac consequences. Hepatology. 2008;48:1570-1576.

29. Shovlin CL, Tighe HC, Davies RJ, et al. Embolisation of pulmonary arteriovenous malformations: no consistent effect on pulmonary artery pressure. Eur Respir J. 2008;32:162-169.

30. Shovlin CL, Sodhi V, McCarthy A, et al. Estimates of maternal risks of pregnancy for women with hereditary haemorrhagic telangiectasia (Osler-Weber-Rendu syndrome): suggested approach for obstetric services. BJOG. 2008;115:1108-1115.

31. Goussous T, Haynes A, Najarian K, et al. Hereditary hemorrhagic telangiectasia presenting as high output cardiac failure during pregnancy. Cardiol Res Pract. 2009;2009:437237.

32. Faughnan ME, Palda VA, Garcia-Tsao G, et al. International guidelines for the diagnosis and management of hereditary hemorrhagic telangiectasia. J Med Genet. 2011;48:73-87.

33. Caselitz M, Bahr MJ, Bleck JS, et al. Sonographic criteria for the diagnosis of hepatic involvement in hereditary hemorrhagic telangiectasia (HHT). Hepatology. 2003;37:1139-1146.

34. DeLeve LD, Valla DC, Garcia-Tsao G. American association for the study liver diseases. Vascular disorders of the liver. Hepatology. 2009;49:1729-1764.

35. Dupuis-Girod S, Chesnais AL, Ginon I, et al. Long-term outcome of patients with hereditary hemorrhagic telangiectasia and severe hepatic involvement after orthotopic liver transplantation: a single-center study. Liver Transpl. 2010;16:340-347.

36. Colli A, Fraquelli M, Andreoletti M, et al. Severe liver fibrosis or cirrhosis: accuracy of US for detection-analysis of 300 cases. Radiology. 2003;227:89-94.

37. Buscarini E, Buscarini L, Civardi G, et al. Hepatic vascular malformations in hereditary hemorrhagic telangiectasia: imaging findings. AJR. 1994;163:1105-1110.

38. Buscarini E, Buscarini L, Danesino C, et al. Hepatic vascular malformations in hereditary hemorrhagic telangiectasia-Doppler sonographic screening in a large family. J Hepatol. 1997;26: 111-118.

39. Buscarini E, Danesino C, Plauchu H, et al. High prevalence of hepatic focal nodular hyperplasia in subjects with hereditary hemorrhagic telangiectasia. Ultrasound Med Biol. 2004;30: 1089-1097.

40. ESC guidelines for the diagnosis and treatment of acute and chronic heart failure 2008. The task force for the diagnosis and treatment of acute and chronic heart failure 2008 of the European Society of Cardiology. Eur Heart J. 2008;29:2388-2442.

41. Garcia-Tsao G. Liver involvement in hereditary hemorrhagic telangiectasia (HHT). J Hepatol. 2007;46:499-507.

42. Blewitt RW, Brown CM, Wyatt JI. The pathology of acute hepatic disintegration in hereditary hemorrhagic telangiectasia. Histopathology. 2003;42:265-269. 
43. Bueres Dominguez I, Annet L, Waignein F, et al. Extensive ischemic liver necrosis complicating hereditary hemorrhagic telangiectasia: a rare indication for liver transplantation. Liver Int. 2005;25:677-679.

44. Mc Inroy B, Zajko AB, Pinna AD. Biliary necrosis due to hepatic involvement with hereditary hemorrhagic telangiectasia. AJR. 1998;170:413-415.

45. Buscarini E, Gebel M, Ocran K, et al. Interobserver agreement in diagnosing liver involvement in hereditary hemorrhagic telangiectasia by Doppler ultrasound. Ultrasound Med Biol. 2008;34: 718-725.

46. Buonamico $P$, Suppressa $P$, Lenato GM, et al. Liver involvement in a large cohort of patients with hereditary hemorrhagic telangiectasia: echo-color Doppler versus multislice CT study. J Hepatol. 2008;48:811-820.
47. Garcia-Tsao G, Sanyal AJ, Grace ND, Carey W, The Practice Guidelines Committee of the American Association for the Study of Liver Diseases, the Practice Parameters Committee of the American College of Gastroenterology. Prevention and management of gastroesophagealvarices and variceal hemorrhage in cirrhosis. Hepatology. 2007;46:922-937.

48. Mitchell A, Adams LA, MacQuillan G, et al. Bevacizumab reverses need for liver transplantation in hereditary hemorrhagic telangiectasia. Liver Transpl. 2008;14:210-213.

49. Buscarini E, Manfredi G, Zambelli A. Bevacizumab to treat complicated liver vascular malformations in hereditary hemorrhagic telangiectasia: a word of caution. Liver Transpl. 2008;14: $1685-1686$ 